\title{
Mammalian RAFT1 kinase domain provides rapamycin-sensitive TOR function in yeast
}

\author{
Clara M. Alarcon, ${ }^{1,3}$ Maria E. Cardenas, ${ }^{1}$ and Joseph Heitman ${ }^{1-4}$ \\ Departments of Genetics ${ }^{1}$ and Pharmacology, ${ }^{2}$ Howard Hughes Medical Institute, ${ }^{3}$ Duke University Medical Center, \\ Durham, North Carolina 27710
}

\begin{abstract}
In complex with the prolyl isomerase FKBP12, the natural product rapamycin blocks signal transduction in organisms as diverse as yeast and man. The yeast targets of FKBP12-rapamycin, TOR1 and TOR2, are large proteins with homology to lipid and protein kinases. A mammalian FKBP12-rapamycin binding protein, RAFT1, shares $39 \%$ and $43 \%$ identity with TOR1 and TOR2 proteins, respectively but has not been linked to rapamycin action in vivo. We find that when expressed in yeast, neither wild-type nor mutant RAFT1 complemented tor mutations or conferred rapamycin resistance. In contrast, TOR2-RAFT1 and TOR1-RAFT1 hybrid proteins containing the carboxy-terminal RAFT1 kinase domain complemented tor2 and tor 1 mutant strains, respectively. Moreover, TOR2-RAFT1 and TOR1-RAFT1 hybrid proteins mutated at the position corresponding to rapamycin-resistant TOR mutants (S2035I) conferred rapamycin resistance. Like the TOR2 protein, the TOR2-RAFT1 proteins were stably expressed, localized to the vacuolar surface, and associated with a phosphatidylinositol-4 kinase activity. These findings directly link the mammalian TOR homolog RAFT1 to rapamycin action in vivo and indicate that the TOR/RAFT1 kinase domain has been functionally conserved from yeast to man.
\end{abstract}

[Key Words: Signal transduction; immunosuppression; prolyl isomerases; RAFT1 kinase domain; TOR; yeast; rapamycin]

Received October 13, 1995; revised version accepted December 7, 1995.

The natural product rapamycin has potent antifungal and immunosuppressive activities through its ability to form toxic complexes with the FKBP12 prolyl isomerase (for review, see Schreiber 1991; Cardenas et al. 1994). The FKBP12-rapamycin complex inhibits signal transduction events required for $G_{1}$ to $S$-phase cell cycle progression in yeast, in $\mathrm{T}$ lymphocytes stimulated with interleukin 2 (IL-2), and in additional mammalian cell types stimulated by a variety of different growth factors (Dumont et al. 1990; Heitman et al. 1991a; Albers et al. 1993; Morice et al. 1993; de Groot et al. 1994). Rapamycin is currently in clinical trials as an immunosuppressant in organ transplant recipients.

Although rapamycin is structurally related to FK506, another immunosuppressive antifungal compound that also binds FKBP12, rapamycin does not inhibit the protein phosphatase calcineurin, which is the target of the FKBP12-FK506 complex (Liu et al. 1991). The targets of the FKBP12-rapamycin complex were first identified in yeast as TOR1 and TOR2 (Heitman et al. 1991a), 245$\mathrm{kD}$ proteins that share $67 \%$ identity and have a carboxyterminal domain with similarity to lipid and protein kinases (Cafferkey et al. 1993; Kunz et al. 1993; Helliwell et al. 1994). TOR2 is an essential protein (Kunz et al. 1993), whereas TOR1 is not (Cafferkey et al. 1993; Hel-

\footnotetext{
${ }^{4}$ Corresponding author.
}

liwell et al. 1994), suggesting that inhibition of TOR2 function by FKBP12-rapamycin is responsible for rapamycin toxicity. However, cells depleted of TOR2 arrest randomly in the cell cycle, whereas those depleted of both TOR 1 and TOR2 arrest in $\mathrm{G}_{1}$, indicating that the $G_{1}$ cell cycle arrest imposed by rapamycin involves inhibition of both TOR proteins (Kunz et al. 1993).

The observation of nonallelic noncomplementation between TOR1, TOR2, and FKBP12 mutants led to the model that FKBP12-rapamycin binds to and inhibits the TOR proteins (Heitman et al. 1991a), which has now been confirmed both in vitro and in vivo (Stan et al. 1994; Cardenas and Heitman 1995; Lorenz and Heitman 1995; Zheng et al. 1995|. The observations that rapamycin caused a similar cell cycle arrest in yeast and man, that yeast and human FKBP12 are 54\% identical and have virtually superimposable tertiary structures (Van Duyne et al. 1991; Rotonda et al. 1993), and that human FKBP12 is functional in yeast (Koltin et al. 1991) all suggested that the TOR proteins would be conserved from yeast to man. An FKBP12-rapamycin binding protein (RAFT1, FRAP, RAPT1, SEP, or mTOR) with marked similarity to TOR1 and TOR2 has been identified (Brown et al. 1994; Chiu et al. 1994; Sabatini et al. 1994; Chen et al. 1995; Sabers et al. 1995). However, evidence linking this mammalian FKBP12-rapamycin binding protein to rapamycin action in vivo has been limited. 
Alarcon et al.

We demonstrate here that whereas full-length RAFT1 failed to provide TOR function in yeast, hybrid TOR2RAFT1 proteins containing the amino-terminal twothirds of TOR2 fused to the RAFT1 carboxy-terminal kinase domain function as TOR2 in yeast. Moreover, S2035I TOR2-mRAFT1 and S2035I TOR1-mRAFT1 mutant proteins rendered yeast rapamycin-resistant; importantly, this mutation corresponds to previously identified rapamycin-resistant TOR1 and TOR2 mutations. Finally, we show that the TOR2-RAFT1 fusion proteins are stably expressed and, as is the case with a TOR2, associated with the surface of the vacuole and PI-4 kinase activity. Our findings directly link the mammalian RAFT1 protein to rapamycin-sensitive growth control in vivo and suggest that RAFT1 performs similar functions in mammalian cells.

\section{Results}

Full-length RAFT1 does not provide TOR2 or TOR1 function

The mammalian FKBP12-rapamycin binding protein, RAFT1, shares $39 \%$ and $43 \%$ overall identity with the yeast TOR 1 and TOR 2 proteins, respectively (Sabatini et al. 1995). We tested whether RAFT1 functions as TOR2 or TOR1 in yeast. A plasmid expressing RAFT1 from the yeast GAL1 promoter (pGAL-RAFT1) was introduced into a diploid yeast strain in which one copy of the TOR2 gene had been disrupted (TOR2/Ator2::LEU2). Tetrad analysis revealed 2 viable: 2 inviable meiotic segregants in all of 60 tetrads examined; in all cases viable segregants were $\mathrm{Leu}^{-}$, indicating that the tor2::LEU2 mutation is not complemented by RAFT1 (data not shown; summarized in Fig. 1). In contrast, expression of the TOR2 gene from the GAL1 promoter restored viability to tor2::LEU2 meiotic segregants (data not shown). These findings suggest that RAFTl does not provide TOR2 function in yeast.

Although TOR 1 is not essential in many yeast strains (Cafferkey et al. 1993; Helliwell et al. 1994), we find that tor1 null mutations are conditionally lethal in srk1 mutant yeast strains at $39^{\circ} \mathrm{C}$ (D. Fiorentino, pers. comm.; $M$. Lorenz and J. Heitman, unpubl.). We therefore tested whether expression of RAFT1 from the yeast GAL1 promoter would complement a tor $1 \Delta s r k 1$ mutant strain (CAY1). Whereas expression of wild-type TOR1 complemented and restored growth of the tor $1 \Delta s r k 1$ mutant strain at $39^{\circ} \mathrm{C}$, RAFTl did not (data not shown; summarized in Fig. 1). These findings suggest that RAFT1 does not provide TORl function in yeast.

Attempts to detect the RAFT1 and mRAFT1 proteins in these complementation assays by Western blot anal-
Figure 1. Structures and phenotypes of TOR-RAFT1 hybrid genes. The wild-type TOR2, TOR1, and RAFT1 genes, the S2035I RAFT1 mutant (mRAFT1), and the TOR2-TOR1, TOR2-RAFT1, TOR2-mRAFT1 (S2035I), TOR1-RAFT1, and TOR1-MRAFT1 (S2035I) hybrid genes are linearly depicted with the amino and carboxyl termini of the corresponding protein product at the left and right, respectively. Sequences derived from TOR2 (solid bars), TOR1 (hatched bars), and RAFT1 (open bars) are indicated. $B$ and $M$ indicate the BamHI and MluI sites, which served as the junctions in the construction of gene fusions. The asterisk $\left({ }^{*}\right)$ represents the S2035I mutation. Complementation and resistance, and failure to complement and drug sensitivity are indicated by + and -, respectively, and are summarized from the experiments presented in Figs. 3 and 4; data not shown. ${ }^{\text {T}}$ The TORl-RAFTl and TOR1-mRAFT1 proteins partially complemented a $\Delta$ tor 1 mutation, and the S2035I TOR1-mRAFTI mutant protein conferred rapamycin resistance when expressed from a $2 \mu$ plasmid but not from a centromeric plasmid.

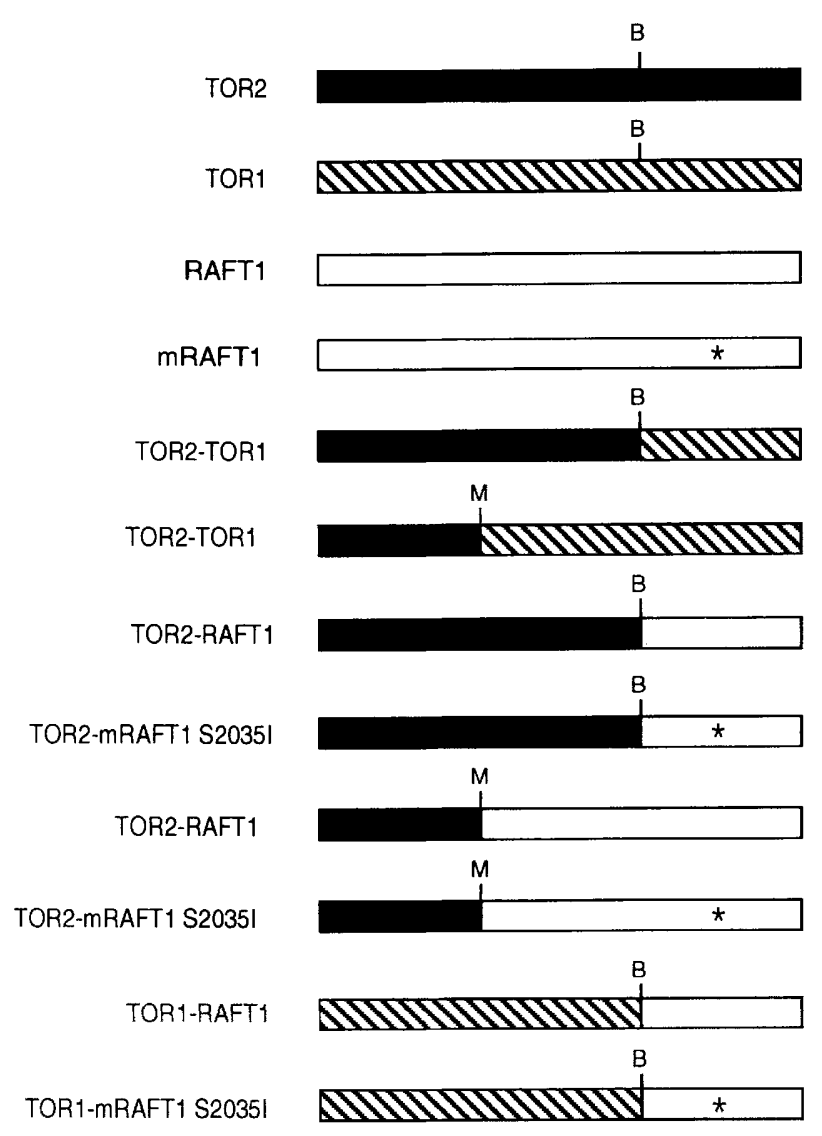

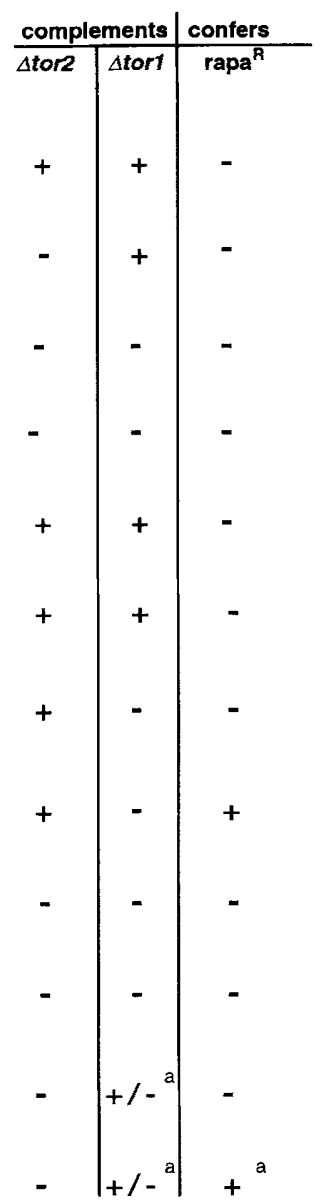


ysis using both antisera and affinity-purified antibodies directed against three different RAFT1 peptides (peptides 64, 782, carboxyl terminus; for more details, see Materials and methods; Sabatini et al. 1995) were unsuccessful (data not shown). Note that these antisera were shown previously to recognize (in Western blots) RAFT1 from rat brain or tissue culture cells (Sabatini et al. 1995). However, by Northern blot analysis, an $\sim 8-\mathrm{kb}$ message corresponding to the RAFT1 mRNA is stably expressed in yeast cells grown under inducing conditions $(+$, Fig. 2$)$ and is absent under repressing conditions $(-$, Fig. 2). These findings suggest that the RAFT1 protein may be unstable or unable to provide TOR1 or TOR2 function in yeast. A failure of RAFT1 to provide TOR function in yeast would not be unanticipated, given that although the yeast TOR 1 and TOR 2 proteins are highly related $(67 \%$ identity $)$, neither complements for the other under standard conditions (Helliwell et al. 1994). Even when overexpressed, only TOR 2 can provide TOR 1 function, whereas TOR1 does not provide TOR2 function (Helliwell et al. 1994).

TOR2-RAFT1 hybrids provide TOR2 function in yeast The highest degree of identity $(\sim 60 \%)$ between RAFT1

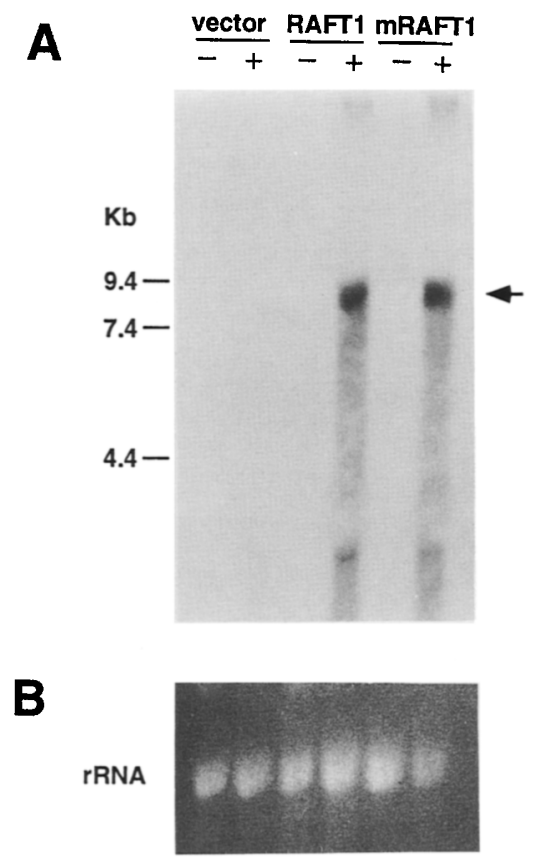

Figure 2. Northern blot analysis of RAFT1 and mRAFT1 expression in yeast. Total RNA was extracted from the TOR1 TOR2 wild-type strain JK9-3d containing the control plasmid pSEYC68 (vector), or the pGAL-RAFT1 (RAFT1) and the pGAL-S2035I-mRAFT1 (mRAFT1) expression plasmids and grown under repressing $(-$, glucose) or inducing $(+$, galactose) conditions. RNA was electrophoresced in a $1 \%$ gel and transferred to a nitrocellulose filter and hybridized with a ${ }^{32} \mathrm{P}$-radiolabeled probe derived from the RAFT1 gene $(A)$ (see Materials and methods) or stained with ethidium bromide to reveal rRNA (B). Numbers at left designate the migration position of molecular weight size standards; the arrow at right indicates the position of migration of the RAFT1 mRNA. and the TOR proteins is in a carboxy-terminal domain similar to the catalytic domains of phosphatidylinositol (PI) and protein kinases. Previous studies demonstrated that the kinase domains of TOR1 and TOR2 are functionally interchangeable (Helliwell et al. 1994). Therefore, we tested whether the kinase domain of RAFTl could functionally replace those of the yeast TOR proteins. For this purpose, hybrid genes were constructed in a CEN LEU2 plasmid in which the promoter region and the amino-terminal two-thirds of TOR2 (residues 1-1687) were fused to the carboxy-terminal one-third of TOR1 (residues 1681-2470) or RAFT1 (residues 17212549 ), and the promoter region and amino terminal twothirds of TOR 1 (residues 1-1680) were fused to the carboxy-terminal one-third of RAFT1 (residues 1721-2549). In addition, TOR2-mRAFT1 and TOR1-mRAFT1 mutant hybrid genes were also constructed in which Ser2035 of RAFT1 was mutated to isoleucine, corresponding to TOR 1 and TOR2 mutations that confer rapamycin resistance. The structures of these hybrid proteins are depicted in Figure 1.

Wild-type TOR2 and TOR1, and the TOR2-TOR1, TOR2-RAFT1, TOR2-mRAFT1, TOR1-RAFT1, and TOR1-mRAFT1 S2035I hybrid proteins (Fig. 1) were tested for their ability to complement and restore viability in a tor2 mutant strain. Low-copy-number centromeric plasmids expressing these proteins were introduced into a tor 2 mutant strain containing the wild-type TOR2 gene on a URA3 plasmid ( $\triangle$ tor 2::ADE2 $\triangle a d e[\mathrm{p} 2 \mu-$ TOR2-URA3) strain MH346-1al. Leu ${ }^{+}$transformants were subjected to a plasmid shuffle on 5-fluoro-orotic acid (5-FOA) medium to eject the resident wild-type TOR2 plasmid and reveal the phenotype conferred by the introduced plasmid. In this assay only those cells expressing a functional TOR2 gene from the introduced plasmid survive. As shown in Figure 3B, wild-type TOR2, and the TOR2-TOR1, TOR2-RAFT1, and TOR2-mRAFT1 hybrid proteins were functional and complemented the tor2 deleted strain; TOR1, TOR1RAFT1, and TOR1-mRAFT1 did not. tor2 mutant cells expressing TOR2-TOR1 or TOR2-RAFT1 fusion proteins grew at the same rate as isogenic wild-type TOR2 cells, were not cold or heat sensitive (data not shown), and were fully rapamycin sensitive (see below, Fig. 3D), indicating that the hybrid proteins provide TOR2 activity that, by these measures, is indistinguishable from wild type. Furthermore, the rapamycin sensitivity of tor2 mutant cells expressing TOR2-RAFT1 required FKBP12 (data not shown).

\section{TOR1-RAFT1 hybrids provide partial TOR1 function}

TOR2, TOR1, and the TOR2-TOR1, TOR1-RAFT1, TOR1-mRAFT1, TOR2-RAFT1, and TOR2-RAFT1 hybrid proteins were then tested for TOR1 function by assaying for rescue of the conditional growth defect of a tor 1 ssrk 1 mutant strain at $39^{\circ} \mathrm{C}$. As shown in Figure $3 \mathrm{C}$, wild-type TOR1, TOR2, and the TOR2-TOR1 hybrid proteins all complemented the tor 1 mutation and restored growth at $39^{\circ} \mathrm{C}$. These observations are in accord 
with previous studies in which increased expression of wild-type TOR2, or of a TOR2-TOR1 hybrid protein, complemented the slow growth phenotype of a tor1 mutant (Helliwell et al. 1994). In contrast, the TOR1RAFT1 and TOR2-RAFT1 hybrid proteins failed to complement the tor 1 mutation when expressed from a centromeric plasmid (Fig. 3C), even though the TOR2RAFT1 hybrid proteins clearly have TOR2 function (Fig. 3B) and are expressed at equivalent levels compared to endogenous TOR2 based on Western blot analysis (Fig. 5, below). In the case of the TOR1-RAFT1 hybrid, expression from a multicopy $2 \mu$ plasmid partially complemented the conditional lethality of a tor $1 \Delta$ srk1 mutant, indicating that the TOR1-RAFT1 protein provides only partial TORl function (Fig. 1; data not shown). The failure of the TOR1-RAFT1 hybrid to provide full TOR1 function could be attributable, at least in part, to the significantly lower level of expression of the hybrid protein compared with the wild-type TOR1 protein (Fig. 4B). In summary, we conclude that the RAFTl kinase domain provides TOR2 activity when fused to TOR2 and provides partial TORl activity when fused to TOR1.

\section{S2035I TOR2-RAFT1 and S2035I TOR1-RAFT1 hybrid proteins confer dominant rapamycin resistance}

The FKBP12-rapamycin complex is toxic in yeast and mutations in a conserved serine residue of TOR1 or TOR2 confer rapamycin resistance (Heitman et al. 1991a; Cafferkey et al. 1993; Helliwell et al. 1994). This serine residue is also conserved in RAFT1 (S2035); therefore, we investigated whether the corresponding RAFT1 mutation would confer rapamycin resistance in yeast. Expression of the full-length S2035 mRAFT1 protein failed to confer rapamycin resistance (Fig. 1). We there-

Figure 3. TOR2 and TOR1 activity and rapamycin resistance conferred by TOR-RAFT hybrid proteins. $(A)$ Isogenic strains expressing wild-type TOR2 and TOR1, and the TOR2-TOR1, TOR2-RAFT1, TOR2-mRAFT S2035I, TOR1-RAFT1, and TOR1-mRAFT S2035I hybrid proteins, were grown on medium as indicated and assayed for TOR2 function, TOR1 function, and rapamycin resistance. $(B)$ Complementation of TOR2 function. Strain MH346-1a, a $\triangle$ tor2::ADE2 strain hosting a TOR2 CEN URA3 plasmid, was transformed with the CEN LEU2 plasmids expressing wild-type and mutant TOR and TOR-RAFT1 hybrid proteins depicted in $A$. Transformants were grown on medium without (-FOA) or with (+FOA) 5-FOA to select against the TOR2 URA3 plasmid and determine whether TOR2 function is provided by the wild-type or hybrid gene introduced. Survival on FOA indicates TOR2 function. $(C)$ Complementation of TOR1 function. A tor $1 \Delta s r k 1$ yeast strain (CAY1) transformed with centromeric plasmid-borne wild-type and mutant TOR and TOR-RAFT1 hybrid genes was grown for $72 \mathrm{hr}$ at $30^{\circ} \mathrm{C}$ and $39^{\circ} \mathrm{C}$. Cells with TOR 1 function survive at $39^{\circ} \mathrm{C}$, whereas cells lacking TOR1 function do not. $(D)$ Rapamycin effects on TOR-RAFT1 hybrids. A TOR1 TOR2 wild-type yeast strain (JK9-3da) transformed with centromeric plasmids expressing wild-type TOR and TOR-RAFT1 hybrid proteins was grown for $72 \mathrm{hr}$ at $30^{\circ} \mathrm{C}$ on medium without (- RAP) or with (+ RAP) 10 $\mu \mathrm{g} / \mathrm{ml}$ of rapamycin. fore introduced the S2035I mutation into the TOR2RAFT1 and TOR1-RAFT1 hybrid proteins. As tested by the tor2 complementation assay, the TOR2-mRAFT1 S2035I hybrid protein fully restored TOR2 activity to tor2 deleted cells (Fig. 3B). Whereas growth of wild-type TOR1 TOR2 yeast cells expressing full-length RAFT1, or the TOR1-RAFT1 or TOR2-RAFT1 hybrid proteins, was inhibited by rapamycin, the TOR2-mRAFT1 S2035I mutant protein conferred resistance up to $10 \mu \mathrm{g} / \mathrm{ml}$ of rapamycin (Fig. 3D). Moreover, because rapamycin resistance was observed in a yeast strain also expressing wildtype TOR2, the TOR2-mRAFT1 hybrid protein confers dominant drug resistance. Finally, the TOR2-mRAFT1 protein conferred rapamycin resistance at $24,30,37$, or

A

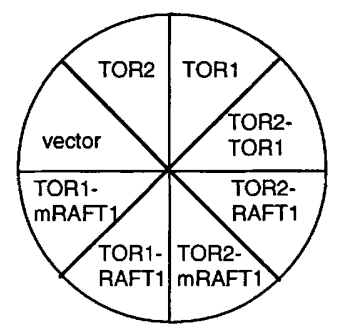

B

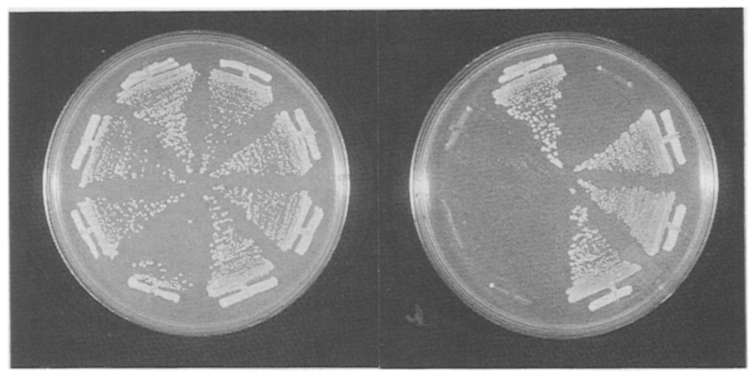

C $30^{\circ} \mathrm{C}$ $39^{\circ} \mathrm{C}$

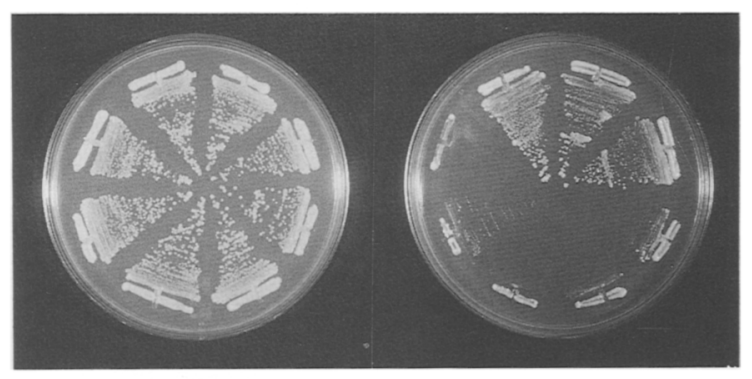

D - RAP + RAP

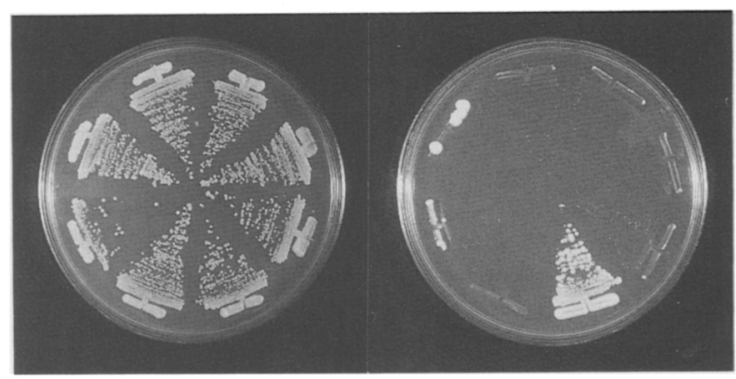




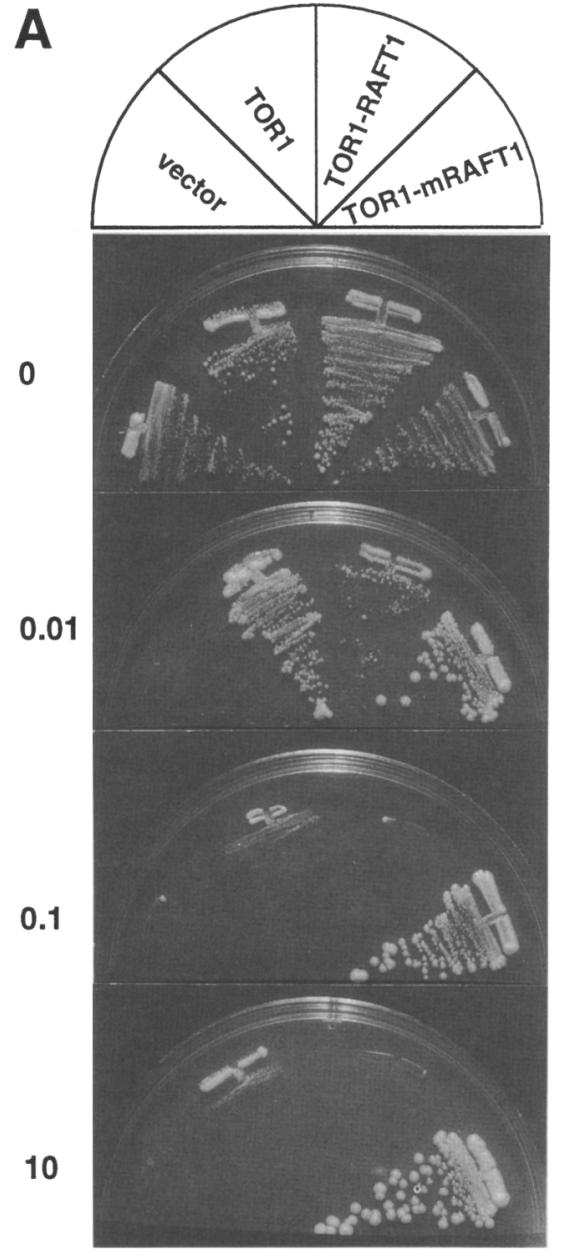

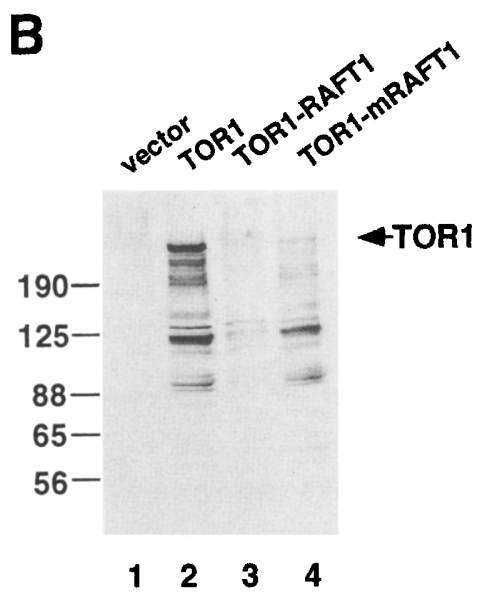

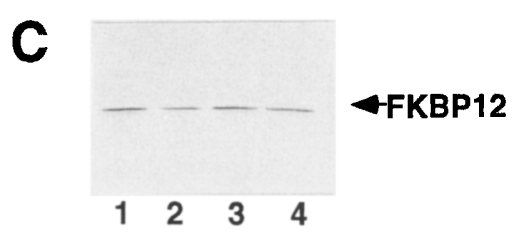

Figure 4. Rapamycin resistance and expression of TOR1-RAFT hybrid proteins. $(A)$ Rapamycin effects on TOR1RAFT1 hybrid proteins. A tor1 TOR2 yeast strain (MLY10a) lacking TOR1 (vector) or expressing TORl (TORl), or the TOR1-RAFT1 wild-type protein (TOR1-RAFT1) or the S2035I TOR1mRAFTl mutant protein (TORlmRAFTl) from the $2 \mu$ URA3 vector pRS306-2 $\mu$ were grown for $72 \mathrm{hr}$ at $39^{\circ} \mathrm{C}$ on medium without $\{0\rangle$ or with $0.01,0.1$, or $10 \mu \mathrm{g} / \mathrm{ml}$ of rapamycin. $(B)$ Expression of TOR1-RAFT1 hybrid proteins. Total cell extracts from strain MLY10a (tor 1::LEU2 TOR2) transfromed with the $2 \mu$, URA3 plasmid pRS306-2 $\mu$ (lane 1) or with the same plasmid bearing the wild-type TOR1 gene (lane 2, TOR1) or the TOR1 hybrid genes, TOR1-RAFT1 (lane 3) and S2035I TOR1-MRAFT1 (lane 4)were prepared. Western blot analysis with $60 \mu \mathrm{g}$ of protein was performed using the anti-TOR 1 antiserum. Note that the specificity of the anti-TOR 1 antiserum is shown by the absence of cross-reacting material in the tor 1 strain transformed with the control plasmid alone (lane 1). In $C, 30 \mu \mathrm{g}$ of protein from the same extracts in $B$ was analyzed by Western blot for FKBP12 to verify that equal amounts of protein were loaded. In $B$ and $C$, numbers at left indicate molecular mass (in $\mathrm{kD}$ ) and the migration positions of TOR1, TOR1-RAFT1, and FKBP12 are indicated. $39^{\circ} \mathrm{C}$, indicating that the mutant fusion protein is not temperature sensitive (data not shown).

Similarly, expression of the TOR1-mRAFT1 mutant hybrid protein conferred rapamycin resistance in a tor 1 TOR2 strain (Fig. 4A) or in a TOR1 TOR2 strain /data not shown|. The ability of the TOR1-mRAFT1 mutant protein to confer rapamycin resistance was observed only with expression from a multicopy $2 \mu$ plasmid but not from a low-copy-number centromeric plasmid (Figs. 3D and 4A). Rapamycin resistance was observed when cells were grown at $24^{\circ} \mathrm{C}-39^{\circ} \mathrm{C}$ (Fig. $4 \mathrm{~A}$ ), indicating that at least some of the mutant protein must be expressed at these temperatures. Western blot analysis with a TOR1specific antiserum revealed that in comparison to wildtype TOR1, a smaller proportion of the TOR1-RAFT1 and TOR1-mRAFTl hybrid proteins were expressed as full-length protein (Fig. 4B). Thus, instability of the TOR1-RAFT1 hybrid proteins is a plausible explanation for the failure of the proteins to provide complete TOR1 function; the fact that rapamycin resistance required a higher level of expression of the mutant protein supports this view. Taken together, these findings directly link the activity of the mammalian RAFTl protein to FKBP12-rapamycin-sensitive growth regulation in vivo.

\section{TOR2-RAFT1 hybrid proteins are expressed as $\sim 240-k D$ proteins}

We have generated a polyclonal antiserum against a TOR2 carboxy-terminal fragment encompassing amino acids 2270-2474. Because TOR1, TOR2, and RAFT1 share a high degree of identity in this region /residues 2270-2370 of TOR2 and the corresponding regions of RAFT1 and TOR 1 share $80 \%$ and $97 \%$ identity, respectively), we considered it likely that the anti-TOR2 antiserum would cross-react with TOR1 and RAFT1 to allow detection of TOR2-RAFT1 and TOR2-TOR1 hybrid proteins by Western blot analysis. Protein extracts were prepared from cells in which the chromosomal TOR1 and TOR2 genes had been deleted and a plasmid-borne TOR2 gene was ejected from the cell and replaced, via plasmid shuffle, with plasmids encoding TOR2 (as a control) or the TOR2-TOR1, TOR2-RAFT1, and TOR2mRAFT1 hybrids. As shown in Figure 5, the TOR2TOR1, TOR2-RAFT1, and TOR2-mRAFT1 hybrid proteins were readily detectable as proteins of $\sim 240 \mathrm{kD}$ that were expressed at a level comparable to the endogenous chromosomal TOR2 gene (Fig. 5, cf. lane 1 with 2-5). This finding is in full accord with our in vivo observa- 


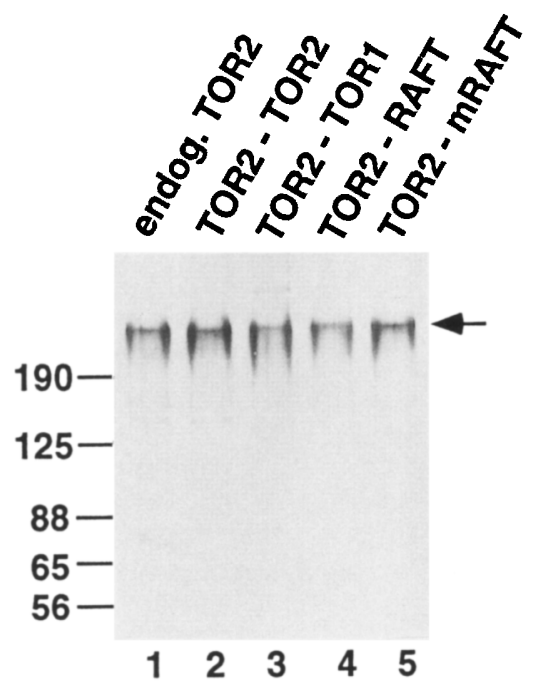

Figure 5. Expression of TOR2-TOR1 and TOR2-RAFT1 hybrid proteins. Total cell extracts from strain MLY10a (tor1::LEU2 TOR2) (lane 1, endog. TOR2), and from strain CAY3 (tor2::ADE2 tor $1 \triangle:: U R A 3$ ) harboring the CEN LEU2 plasmid pRS315 expressing the wild-type TOR2 protein (lane 2, TOR2-TOR2) or the TOR2 hybrid proteins TOR2-TOR1 (lane 3), TOR2-RAFT1 (lane 4), and the TOR2-mRAFT1 S2035I mutant (lane 5) were prepared. From these extracts, $60 \mu \mathrm{g}$ of protein was fractionated by SDS-PAGE and analyzed by Western blot with the anti-TOR2 carboxy-terminal antiserum. The arrow indicates the migration position of TOR2 and the TOR2 hybrid proteins. Numbers at left indicate molecular mass (in kD).

tions that the TOR2-TOR1, TOR2-RAFT1, and the TOR2-mRAFT1 hybrid proteins rescue tor2 mutant cells, and that the TOR2-mRAFT1 mutant protein confers rapamycin resistance.

\section{TOR2-TOR1 and TOR2-RAFT1 hybrid proteins are localized to the vacuolar membrane}

We have demonstrated previously that the TOR2 protein is membrane associated with and based on immunofluorescence and cellular fractionation, localized to the vacuolar membrane (Cardenas and Heitman 1995). By light microscopy and Nomarski imaging, yeast cells lacking TOR1 and TOR2 and surviving solely by expression of the TOR2-TOR1, TOR2-RAFT1, or TOR2-mRAFT1 hybrid proteins had no grossly apparent morphological defects and contained normal vacuoles (data not shown). Indirect immunofluorescence with an antiserum that recognizes an amino-terminal TOR2 peptide revealed that as is the case with the wild-type TOR2 protein, the TOR2-TOR1, TOR2-RAFT1, and TOR2-mRAFT1 hybrid proteins were similarly localized to the surface of the yeast vacuole (data not shown).

\section{TOR2-TOR1, TOR2-RAFT1 hybrids have associated PI-4 kinase activity}

Immunoprecipitates of both TOR2 (Cardenas and Heitman 1995) and RAFT1 (Sabatini et al. 1995) contain PI-4 kinase activity. To determine whether TOR2-RAFT1 hybrid proteins have a similar associated activity, wildtype TOR2 and the TOR2-TOR1, TOR2-RAFT1, and TOR2-mRAFT1 hybrid proteins were immunoprecipitated with the anti-TOR2 carboxy-terminal antiserum that effectively cross-reacts with TOR2, TOR1, and RAFT1 (Fig. 5). In addition, the proteins were obtained from cells in which the chromosomal TOR1 and TOR2 genes had been disrupted and only a plasmid-borne TOR2, TOR2-TOR1, or TOR2-RAFT1 hybrid gene was present. The resulting immunoprecipitates were assayed for PI kinase activity by incubation with $\left[\gamma^{32}\right.$ P]ATP. PI kinase reactions were resolved by thin layer chromatography in the borate system (Walsh et al. 1991) to resolve PI3P and PI4P. As described previously for the TOR 2 and RAFT1 proteins (Cardenas and Heitman 1995; Sabatini et al. 1995), a readily detectable PI-4 kinase activity was present in immunoprecipitates of the TOR2-TOR1, TOR2-RAFT1, and TOR2-mRAFT1 fusion proteins (Fig. 6). Moreover, the PI-4 kinase activity detected was at a level comparable to that associated with the endogenous TOR2 protein immunoprecipitated in parallel. These findings reveal that a PI- 4 kinase activity is tightly associated with and coimmunoprecipitates with the TOR2-TOR1 and TOR2-RAFT1 hybrid proteins.

\section{Discussion}

When bound to the peptidyl-prolyl isomerase FKBP12, the immunosuppressive antifungal natural product rapamycin inhibits signaling cascades required for growth and cell cycle progression, which are conserved from yeast to man. Genetic studies in yeast first implicated TOR1 and TOR2 as targets of the toxic FKBP12-rapamycin complex (Heitman et al. 1991a; Kunz et al. 1993). Both wild-type TOR1 and TOR2 directly interact with FKBP12-rapamycin whereas rapamycin-resistant TOR1 and TOR2 mutant proteins do not (Stan et al. 1994; Cardenas and Heitman 1995; Lorenz and Heitman 1995; Zheng et al. 1995). The resulting model is that the essential yeast TOR2 protein is required for events necessary for $G_{1}$ to $S$-phase progression and cell division, and FKBP12-rapamycin binding to TOR2 inhibits some function necessary to execute these events properly.

A mammalian FKBP12-rapamycin-binding protein with marked identity to the yeast TOR proteins has been identified and is variously referred to as RAFT1, FRAP, RAPTl, SEP, or mTOR; however, evidence linking this protein to rapamycin action in vivo has been limited and indirect (Brown et al. 1994; Chiu et al. 1994; Sabatini et al. 1994; Chen et al. 1995; Sabers et al. 1995). First, the decreased affinity of two FKBP12-rapamycin analog complexes (16-keto-rap and 25,26-iso-rap) for the FRAP protein is correlated with decreased in vivo activity (Brown et al. 1994). Second, introduction of mutations corresponding to rapamycin-resistant yeast TOR 1 and TOR2 mutations reduces FKBP12-rapamycin binding to fragments of FRAP in vitro (Chen et al. 1995) or to a small portion of RAPT1 or RAFT1 in the two-hybrid in vivo assay (Chiu et al. 1994; Lorenz and Heitman 1995). 


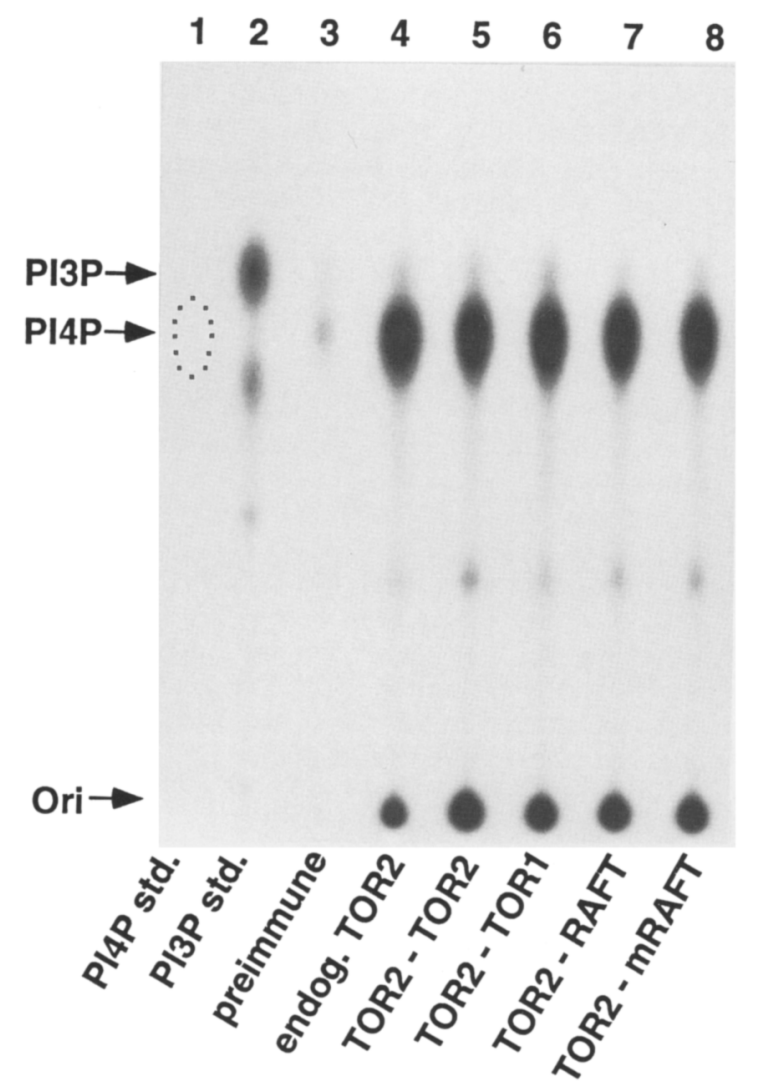

Figure 6. Immunoprecipitates of TOR2-TOR1 and TOR2RAFT1 hybrid proteins exhibit PI-4 kinase activity. Equal amounts of protein extract $(15.5 \mathrm{mg})$ from strain MLY10a (tor 1::LEU2 TOR2) (endog. TOR2, lane 4) or from strain CAY3 (tor2::ADE2 tor1 $\triangle: U U R A 3$ ) expressing from plasmid-borne genes, wild-type TOR2 protein (TOR2-TOR2, lane 5), or the TOR2 hybrid proteins TOR2-TOR1 (lane 6), TOR2-RAFT1 (lane 7), and TOR2-mRAFT1 S2035I mutant (lane 8) were immunoprecipitated with the the anti-TOR2 carboxy-terminal antiserum. The immunoprecipitates were assayed for their ability to phosphorylate phosphatidylinositol, and the reaction products were analyzed by thin layer chromatography using the borate system as described (Walsh et al. 1991). As a control, a cell extract from strain MLY10a (tor1 TOR2) was immunoprecipitated with the preimmune serum (lane 3 ). $\left[{ }^{32} \mathrm{P}\right]$ Phosphatidylinositol-3 phosphate and $\left[{ }^{3} \mathrm{H}\right]$ phosphatidylinositol-4-phosphate (PI3P and PI4P) standards were chromatographed in lanes 1 and 2 , respectively, and their migration positions are indicated by arrows. The origin at which samples were applied is indicated (Ori).

Third, no binding of the mTOR protein to FKBP12-rapamycin affinity matrices was observed with extracts from rapamycin-resistant Jurkat $T$-cell lines (Sabers et al. 1995); however, in this case it is not known whether these mutant cell lines express mTOR, and no mutations in the mTOR locus have been identified. Finally, after this manuscript had been submitted, it was reported that FRAP regulates $p 70 \mathrm{~S} 6$ kinase in human $\mathrm{T}$ cells in a rapamycin-sensitive fashion, linking FRAP to rapamycin action in vivo (Brown et al. 1995). However, in this case, the role of P70 S6 kinase, if any, in growth factor-dependent cellular proliferation remains to be established. An additional concern is that of the two FKBP12-rapamycin-binding proteins identified in yeast, TORl is not essential for growth and is therefore an unlikely target for the major FKBP12-rapamycin toxic effects. Thus, the mammalian FKBP12-rapamycin binding protein that has been identified might be a TOR1 homolog that does not play an essential role in rapamycinsensitive signaling cascades in mammalian cells.

We have assessed the ability of the mammalian FKBP12-rapamycin-binding protein RAFT1 to function in FKBP12-rapamycin sensitive, TOR-dependent cell growth by heterologous expression in the yeast Saccharomyces cerevisiae. Expression of full-length RAFT1 failed to provide either TOR1 or TOR2 function in yeast. Similarly, expression of an S2035I RAFT1 mutant protein, corresponding to rapamycin-resistant TOR mutants, failed to confer rapamycin resistance in yeast. With the caveat that although we detect the RAFT1 mRNA in these cells we have been unable to detect the RAFT1 protein, these findings suggest that full-length RAFT1 lacks some activity required for TOR function in yeast. For example, RAFTl might fail to associate properly with yeast proteins required for TOR function and cellular localization or might not be properly folded, post-translationally modified, or stably expressed in yeast.

As an alternative approach to assess RAFT1 function in yeast, we constructed TOR2-RAFT1 and TOR1RAFT1 hybrid proteins in which the carboxy-terminal one-third of the TOR proteins, encompassing the kinase domain, was replaced with the corresponding domain of RAFT1. These experiments were based on the earlier observation that the kinase domains of TOR1 and TOR2 are functionally interchangeable (Helliwell et al. 1994). Remarkably, we find that a TOR2-RAFT1 hybrid protein can functionally replace the yeast TOR2 protein. The TOR2-RAFT1 hybrid protein supports growth that, by several measures, is indistinguishable from that of wild-type cells, including sensitivity to FKBP12-rapamycin and normal growth under a variety of different physiological conditions. Moreover, although some conditions that compromise TOR2 function render TOR1 essential (Cardenas and Heitman 1995), TOR1 is not essential in the TOR2-RAFT1 strain. One distinction is that when expressed from a centromeric plasmid, wildtype TOR2 can substitute for TOR1 (Fig. 3C), but the TOR2-RAFT1 fusion protein does not, even though this fusion protein clearly provides TOR 2 function. In addition, the TOR1-RAFT1 fusion protein provided partial TOR1 function in a TOR1-dependent yeast strain when expressed from a multicopy $2 \mu$ plasmid. As a second measure of RAFT1 function in yeast, we demonstrated that S2035I mutant TOR2-RAFT1 and S2035I TOR1RAFT1 hybrid proteins confer dominant rapamycin resistance in yeast (Figs. 3D and 4A). Finally, we found that like the wild-type TOR2 protein, the TOR2-TOR1 and TOR2-RAFT1 hybrid proteins were both localized to the vacuolar surface and associated with a PI-4 kinase activ- 
ity. Taken together with previous studies (Cardenas and Heitman 1995), these findings reveal a further correlation suggesting that TOR2 activity in vivo requires both proper localization and an associated lipid kinase activity. We note that Sabatini and colleagues have also detected PI-4 kinase activity associated with RAFT1 (Sabatini et al. 1995).

Several recent reports document that kinase inactive mutants of TORl (Zheng et al. 1995; Cardenas and Heitman, unpubl.), TOR2 (Cardenas and Heitman 1995), and FRAP (Brown et al. 1995) fail to complement tor1 or tor2 mutations, to activate P70 S6 kinase in vivo, or to confer rapamycin resistance. These findings indicate that the activity of the kinase domain of TOR proteins is required for biological function. Our studies reveal that the kinase domain of TOR2 and RAFT1 has been functionally conserved from yeast to man and demonstrate that this domain of RAFTl can function in rapamycin-sensitive, TOR2-dependent signaling cascades in yeast. Moreover, our findings demonstrate further that the RAFT1 kinase domain is functionally related to TOR2, mitigating the concerns that RAFT1 might be a nonessential TOR homolog in mammals. Finally, our finding that a mutation corresponding to rapamycin-resistant TOR1 and TOR2 mutations similarly renders TOR2-RAFT1 and TOR1RAFT1 hybrid proteins rapamycin resistant directly links the RAFT1 protein to FKBP12-rapamycin action in vivo. Further studies will be required to test whether the corresponding RAFT1 mutant protein confers rapamycin resistance in mammalian cells responding to growth factors, such as IL-2, and to determine the roles of RAFT1 in rapamycin-inhibitable mammalian signal transduction cascades.

\section{Materials and methods}

Yeast strains

Yeast strains that were employed in this study were all isogenic derivatives of strain JK9-3d (MATa, MAT $\alpha$, or MATa/ $\alpha$ ura3-52 leu2-3,112 trp1-1 his4 rme1 HMLa) (Heitman et al. 1991b), with only changes indicated: MH343, MATa/ $\alpha$ TOR2/ $\triangle$ tor2::LEU2; MH346-la, MATa $\Delta$ tor2::ADE2 $\triangle a d e 2$ (p2 $\mu$-TOR2-URA3); MLY10a, MATa tor1::LEU2 TRP ${ }^{+}$; CAY1, MATa tor $1::$ leu2:: hisG $\Delta$ srk1::G418 TRP ${ }^{+}$; CAY3, MATa tor1 $:: U R A 3$ Itor2::ADE2 $\triangle$ ade2 (pCEN-TOR2, TOR2-TOR1, TOR2-RAFT1, or TOR2mRAFT1 LEU2). Strain CAY1 was obtained from strain MLY10a by one-step gene disnuptions with a PCR product bearing $\Delta s r k 1:: G 418$ obtained with primers 5'-TCCATCTTTATACACTATGTCTAAAAATAGCAACGTTAACAGCTGAAGCTTCGTACGC and 5'-AGATTATACCCTCTTCATGAATGGATTTAATGCACGGACGGCATAGGCCACTAGTGGATCTG (homology to the G418 resistance gene underlined) and template plasmid pFA6-kanMX2 (Wach et al. 1994), and disruption with BgIIcleaved pNKY85 (Alani et al. 1987) and 5-FOA selection to convert tor1::LEU2 to tor1::leu2::hisG. The TOR1 gene was disrupted with BamHI-cleaved pLW1 to yield the tor $1 \Delta:: U R A 3$ strain CAY3 as described (Cardenas and Heitman 1995).

\section{Plasmids}

Plasmids pGAI-RAFT1 (CEN URA3) and pGAL-mRAFT1 S2035I (CEN URA3) express RAFT1 from the GAL1 promoter of plasmid pSEYC68. RAFT1 was PCR amplified from a RAFT1 cDNA (Sabatini et al. 1994) with primers 5'-GCTCTAGAGCGAACCTCAGGGCAAGATGCT and 5'-GCCCAAGCTTCCTTGTGTTTCTGACAGTGT containing $X b a I$ and HindIII sites, respectively, in bold. The $\sim 8-\mathrm{kb}$ PCR product was $X \mathrm{baI}-$ HindIII cleaved and cloned into pSEYC68. mRAFT1 S2035I was created by PCR overlap mutagenesis (Ho et al. 1989) using mutagenic primers 5'-CAAGCGAATGGCCTCTTCTAGGCCTTC and 5'-GAAGAGGCCATTCGCTTGTACTTTGGGGA, and flanking primers 5'-GGTGAAGGCCATACAGATTGACAC (hybridizes $5^{\prime}$ to the unique KpnI site in RAFT1) and 5'GCCCAAGCTTCCTTGTGTTTCTGACAGTGT. This PCR product was $K p n I-H i n d I I I$ cleaved and used to replace the wildtype fragment in pGAL-RAFT1.

Plasmids expressing TOR2 or TOR1 hybrid proteins were constructed as follows. pTOR2-TOR1 was constructed by cleaving plasmid pML40 a derivative of CEN LEU2 plasmid pRS315 (Sikorski and Hieter 1989) bearing an 8-kb NotI fragment containing the TOR2 gene (Lorenz and Heitman 1995) with $\mathrm{BamHI}$, and replacing the excised region with a $2.6-\mathrm{kb}$ $B a m H I$ fragment from plasmid pCNTOR1 [a derivative of CEN URA3 plasmid pRS316 (Sikorski and Hieter 1989) bearing an 8-kb NotI fragment containing theTOR1 gene (Muir and Heitman, unpubl.)].

The TOR2-TOR1 hybrid containing one-third TOR2 and two-thirds TOR1 was constructed by PCR amplification of a segment of TORl using pCNTORl as a template and primers $5^{\prime}$-CCGCACGCGTTATCTTAAGGATTTGTTTCCTTTAA and 5'-CCGCGTCGACGTACCGATGTGCCATCTATCCAAT (MluI and SalI sites shown in bold). The $\sim 5.3-\mathrm{kb}$ PCR product was cloned in the TA cloning system (Invitrogen), excised with MluI-SaII, and subcloned into gapped pML40 that had been digested with MluI-SalI.

pTOR2-RAFT1 was constructed by PCR amplification of the RAFT1 kinase domain with primers 5'-CGGGATCCGGTGCAGACCATGCAGCAGCAG (467) and 5'-GCCCAAGCTTCCTTGTGTTTCTGACAGTGT (411). The $\sim 2.6-\mathrm{kb}$ PCR product was cloned into the TA cloning system (Invitrogen), excised with BamHI, and cloned in the BamHI site of pML40. pTOR2-mRAFT1 S2035I was constructed as pTOR2-RAFT1 except pGAL-mRAFT1 S2035I was used as PCR template. The TOR2-RAFT1 hybrids containing one-third TOR2 and twothirds RAFT1 were constructed similarly to the TOR2-TOR1 hybrid described above using RAFT1 cDNA or PGAL-mRAFT1 as templates and primers 5'-CCGCACGCGTTGGGTGGACGAGCTCTTTGTCATC and 5'-GCACGCGTCGACCTTGTGTTTCTGACAGTGTCT.

pTOR1-RAFT1 and pTOR1-mRAFT1 S2035I were constructed by partially digesting plasmid pCNTOR 1 with $B a m H I$, purifying the linearized plasmid $(12.5 \mathrm{~kb})$, digesting with $\mathrm{XbaI}$, and purifying the BamHI to XbaI-gapped plasmid (10 kb). RAFT1 and mRAFT1 S2035I kinase domains were excised from the TA cloning vector as $B a m H I-X b a I$ fragments and subcloned into the $10-\mathrm{kb}$ pCNTOR 1 gapped plasmid. In addition, TOR1, TOR1-RAFT1, and TOR1-mRAFT were subcloned as NotI cassettes into the CEN LEU2 and $2 \mu$ URA3 plasmids pRS3 15 and pRS306-2 $\mu$, respectively (Sikorski and Hieter 1989).

\section{Northern blot analysis}

Total yeast RNA was isolated by the hot phenol method according to Schmitt et al. (1990). Total RNA was separated on a $1 \%$ agarose gel containing formaldehyde and transferred to a nitrocellulose membrane by the capillary method. Hybridization was performed as described (Sambrook et al. 1989). A RAFT1 PCR fragment using oligonucleotides 467 and 411 (described 
TOR kinase domain conserved from yeast to man

above) was gel purified and radiolabeled using a random priming kit (Boehringer-Mannheim).

\begin{abstract}
Antisera
Generation and characterization of polyclonal antiserum against the TrpE-TOR2 carboxy-terminal fusion protein (used here for Western blots and immunoprecipitation) and aminoand carboxy-terminal TOR2 peptides (used for immunofluorescence) have been described (Cardenas and Heitman 1995).

An anti-TOR 1 antiserum was generated as follows. An amino-terminal fragment of the TOR1 gene encoding amino acids 1-100 was PCR amplified with oligonucleotides 5 'ATATGGATCCCATGGAACCGCATGAGGAGCAG (313) and 5'-CGCGCTGCAGCTACTATAGATCAAATAATGAAATACTGGCC (304). The resulting PCR product was purified, cleaved with BamHI-PstI, and cloned into the BamHI-PstI sites of the TrpE fusion vector pATH22 (Koerner et al. 1991). The TOR1TrpE fusion protein was overexpressed and recovered from inclusion bodies as described (Koemer et al. 1991). For rabbit immunization, the TOR1-TrpE protein was gel purified and electroeluted. Rabbits were injected, boosted, and bled by standard procedures (Harlow and Lane 1988). The anti-FKBP12 polyclonal antiserum has been described previously /Cardenas and Heitman 1995). Antisera and affinity-purified antibodies directed against RAFT1 peptides corresponding to residues 64-79 $(\alpha-64-R A F T 1), 782-797$ ( $\alpha-782-R A F T 1)$, and 2528-2549 ( $\alpha$-carboxy-term-RAFT1) have been described (Sabatini et al. 1995) and were generously provided by David Sabatini and Solomon Snyder (Johns Hopkins University, Baltimore, MD).
\end{abstract}

\section{Yeast cell extracts}

Preparation of yeast cell extracts for Western blot and immunoprecipitation were as described previously (Cardenas and Heitman 1995).

\section{Immunological techniques}

Western blot, immunofluorescence, and immunoprecipitations were performed exactly as described previously (Cardenas and Heitman 1995).

\section{PI kinase assays}

TOR2 immunoprecipitates were assayed for PI kinase activity as described previously (Cardenas and Heitman 1995).

\section{Acknowledgments}

We thank R. Scott Muir for technical assistance, Mike Hall for strains and plasmids, and Mike Lorenz for comments on the manuscript. We are indebted to David Sabatini and Solomon Snyder for generously providing the RAFT1 cDNA and antiRAFTl antisera. This work was supported in part by National Institutes of Health grant (PO1 HL50985-01) and a Council for Tobacco Research grant (4050) to M.E.C. J.H. is an investigator of the Howard Hughes Medical Institute.

The publication costs of this article were defrayed in part by payment of page charges. This article must therefore be hereby marked "advertisement" in accordance with 18 USC section 1734 solely to indicate this fact.

\section{References}

Alani, E., L. Cao, and N. Kleckner. 1987. A method for gene disruption that allows repeated use of URA3 selection in the construction of multiply disruption yeast strains. Genetics 116: $541-545$.

Albers, M.W., R.T. Williams, E.J. Brown, A. Tanaka, F.L. Hall, and S.L. Schreiber. 1993. FKBP-Rapamycin inhibits a cyclindependent kinase activity and a cyclin D1-Cdk association in early Gl of an osteosarcoma cell line. J. Biol. Chem. 268: 22825-22829.

Brown, E.J., M.W. Albers, T.B. Shin, K. Ichikawa, C.T. Keith, W.S. Lane, and S.L. Schreiber. 1994. A mammalian protein targeted by G1-arresting rapamycin-receptor complex. $\mathrm{Na}$ ture 369: 756-759.

Brown, E.J., P.A. Beal, C.T. Keith, J. Chen, T.B. Shin, and S.L. Schreiber. 1995. Control of p70 S6 kinase by kinase activity of FRAP in vivo. Nature 377: 441-446.

Cafferkey, R., P.R. Young, M.M. McLaughlin, D.J. Bergsma, Y. Koltin, G.M. Sathe, L. Faucette, W.-K. Eng, R.K. Johnson, and G.P. Livi. 1993. Dominant missense mutations in a novel yeast protein related to mammalian phosphatidylinositol 3-kinase and VPS34 abrogate rapamycin cytotoxicity. Mol. Cell. Biol. 13: 6012-6023.

Cardenas, M.E. and J. Heitman. 1995. FKBP12-Rapamycin target TOR2 is a vacuolar protein with an associated phosphatidylinositol-4 kinase activity. EMBO J. 14: 5892-5907.

Cardenas, M.E., M. Lorenz, C. Hemenway, and J. Heitman. 1994. Yeast as model-T cells. Perspect. Drug Discovery Design 2: 103-126.

Chen, J., X.-F. Zheng, E.J. Brown, and S.L. Schreiber. 1995. Identification of an 11-kDa FKBP12-rapamycin-binding domain within the 289-kDa FKBP12-rapamycin-associated protein and characterization of a critical serine residue. Proc. Natl. Acad. Sci. 92: 4947-4951.

Chiu, M.I., H. Katz, and V. Berlin. 1994. RAPT1, a mammalian homolog of yeast Tor, interacts with the FBKP12/rapamycin complex. Proc. Natl. Acad. Sci. 91: 12574-12578.

de Groot, R.P., L.M. Ballou, and P. Sassone-Corsi. 1994. Positive regulation of the cAMP-responsive activator CREM by the p70 S6 kinase: An alternative route to mitogen-induced gene expression. Cell 79: 81-91.

Dumont, F.J., M.R. Melino, M.J. Staruch, S.L. Koprak, P.A. Fischer, and N.H. Sigal. 1990. The immunosuppressive macrolides FK-506 and rapamycin act as reciprocol antagonists in murine T-cells. J. Immunol. 144: 1418-1424.

Harlow, E. and D. Lane. 1988. Antibodies: A laboratory manual. Cold Spring Harbor Laboratory, Cold Spring Harbor, NY.

Heitman, J., N.R. Movva, and M.N. Hall. 1991a. Targets for cell cycle arrest by the immunosuppressant rapamycin in yeast. Science 253: 905-909.

Heitman, J., N.R. Movva, P.C. Hiestand, and M.N. Hall. 1991b. FK506-binding protein proline rotamase is a target for the immunosuppressive agent FK506 in Saccharomyces cerevisiae. Proc. Natl. Acad. Sci. 88: 1948-1952.

Helliwell, S.B., P. Wagner, J. Kunz, M. Deuter-Reinhard, R. Henriquez, and M.N. Hall. 1994. TOR1 and TOR2 are structurally and functionally similar but not identical phosphatidylinositol kinase homologues in yeast. Mol. Biol. Cell 5: 105118.

Ho, S.N., H.D. Hunt, R.M. Horton, J.K. Pullen, and L.R. Pease. 1989. Site-directed mutagenesis by overlap extension using the polymerase chain reaction. Gene 77: 51-59.

Koerner, T.J., J.E. Hill, A.M. Myers, and A. Tzagoloff. 1991. High-expression vectors with multiple cloning sites for con- 
struction of $\operatorname{trp} E$ fusion genes: pATH vectors. Methods Enzymol. 194: 477-490.

Koltin, Y., L. Faucette, D.J. Bergsma, M.A. Levy, R. Cafferkey, P.L. Koser, R.K. Johnson, and G.P. Livi. 1991. Rapamycin sensitivity in Saccharomyces cerevisiae is mediated by a peptidyl-prolyl cis-trans isomerase related to human FK506binding protein. Mol. Cell. Biol. 11: 1718-1723.

Kunz, J., R. Henriquez, U. Schneider, M. Deuter-Reinhard, N.R. Movva, and M.N. Hall. 1993. Target of rapamycin in yeast TOR2, is an essential phosphatidylinositol kinase homolog required for $G_{1}$ progression. Cell 73: $585-596$.

Liu, J., J.D. Farmer, W.S. Lane, J. Friedman, I. Weissman, and S.L. Schreiber. 1991. Calcineurin is a common target of cyclophilin-cyclosporin A and FKBP-FK506 complexes. Cell 66: 807-815.

Lorenz, M.C. and J. Heitman. 1995. TOR mutations confer rapamycin resistance by preventing interaction with FKBP12rapamycin. J. Biol. Chem. 270: 27531-27537.

Morice, W.G., G.J. Brunn, G. Wiederrecht, J.J. Siekierka, and R.T. Abraham. 1993. Rapamycin-induced inhibition of p34 ${ }^{\text {cdc2 }}$ kinase activation is associated with $G_{1} / S$-phase growth arrest in T lymphocytes. $J$. Biol. Chem. 268: 3734 3738.

Rotonda, J., J.J. Burbaum, H.K. Chan, A.I. Marcy, and J.W. Becker. 1993. Improved calcineurin inhibition by yeast FBKP12-drug complexes. J. Biol. Chem. 268: 7607-7609.

Sabatini, D.M., H. Erdjument-Bromage, M. Lui, P. Tempst, and S.H. Snyder. 1994. RAFT1: A mammalian protein that binds to FKBP12 in a rapamycin-dependent fashion and is homologous to yeast TORs. Cell 78: $35-43$.

Sabatini, D.M., B.A. Pierchala, R.K. Barrow, M.J. Schell, and S.H. Snyder. 1995. The rapamycin and FKBP12 target (RAFT) displays phosphatidylinositol 4-kinase activity. I. Biol. Chem. 270: 20875-20878.

Sabers, C.J., M.M. Martin, G.J. Brunn, J.M. Williams, F.J. Dumont, G. Wiederrecht, and R.T. Abraham. 1995. Isolation of a protein target of the FKBP12-rapamycin complex in mammalian cells. $J$. Biol. Chem. 270: 815-822.

Sambrook, J., E.F. Fritsch, and T. Maniatis. 1989. Molecular cloning: A laboratory manual. Cold Spring Harbor Laboratory Press, Cold Spring Harbor, NY.

Schmitt, M.E., T.A. Brown, and B.L. Trumpower. 1990. A rapid and simple method for preparation of RNA from Saccharomyces cerevisiae. Nucleic Acids Res. 18: 3091-3092.

Schreiber, S.L. 1991. Chemistry and biology of the immunophilins and their immunosuppressive ligands. Science 251: 283287.

Sikorski, R.S. and P. Hieter. 1989. A system of shuttle vectors and yeast host strains designed for efficient manipulation of DNA in Saccharomyces cerevisiae. Genetics 122: 19-27.

Stan, R., M.M. McLaughlin, R. Cafferkey, R.K. Johnson, M. Rosenberg, and G.P. Livi. 1994. Interaction between FKBP12-Rapamycin and TOR involves a conserved serine residue. I. Biol. Chem. 269: 32027-32030.

Van Duyne, G.D., R.F. Standaert, P.A. Karplus, S.L. Schreiber, and J. Clardy. 1991. Atomic structure of FKBP-FK506, an immunophilin-immunosuppressant complex. Science 252: 839-842.

Wach, A., A. Brachat, R. Pohlmann, and P. Philippsen. 1994. New heterologous modules for classical or PCR- based gene disruptions in Saccharomyces cerevisiae. Yeast 10: 1793 1808.

Walsh, J.P., K.K. Caldwell, and P.W. Majerus. 1991. Formation of phosphatidylinositol 3-phosphate by isomerization from phosphatidylinositol 4-phosphate. Proc. Natl. Acad. Sci. 88: 9184-9187.
Zheng, X.-F., D. Fiorentino, J. Chen, G.R. Crabtree, and S.L. Schreiber. 1995. TOR kinase domains are required for two distinct functions, only one of which is inhibited by rapamycin. Cell 82: 121-130. 


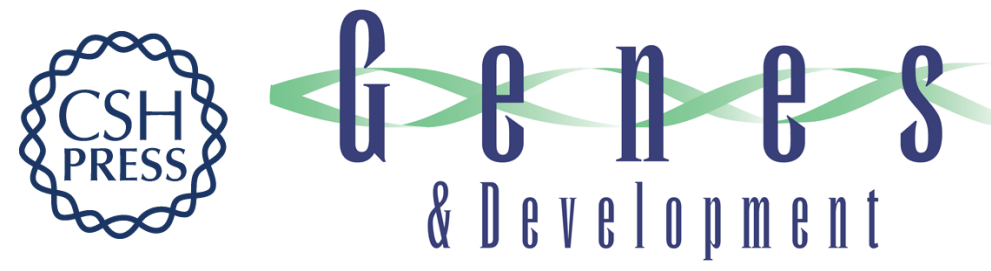

\section{Mammalian RAFT1 kinase domain provides rapamycin-sensitive TOR function in yeast.}

C M Alarcon, M E Cardenas and J Heitman

Genes Dev. 1996, 10:

Access the most recent version at doi:10.1101/gad.10.3.279

References This article cites 33 articles, 20 of which can be accessed free at: http://genesdev.cshlp.org/content/10/3/279.full.html\#ref-list-1

License

Email Alerting

Service

Receive free email alerts when new articles cite this article - sign up in the box at the top right corner of the article or click here.

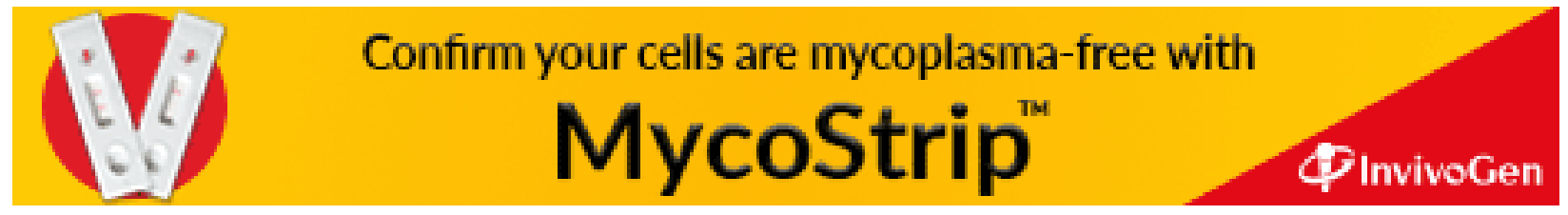

